doi: $10.2306 /$ scienceasia1513-1874.2013.39.257

\title{
Principal component analysis identifies major muscles recruited during elite vertical jump
}

\author{
Nongnapas Charoenpanich $^{\mathrm{a}}$, Rumpa Boonsinsukh ${ }^{\mathrm{b}}$, Sirod Sirisup $^{\mathrm{c}}$, Vitoon Saengsirisuwan $^{\mathrm{a}, *}$ \\ a Department of Physiology, Faculty of Science, Mahidol University, Rama 6 Road, Bangkok 10400 Thailand \\ b Division of Physical Therapy, Faculty of Health Sciences, Srinakharinwirot University, \\ Rangsit-Nakhonnayok Road, Ongkharak, Nakhonnayok 26120 Thailand \\ c Large-Scale Simulation Research Laboratory, National Electronics and Computer Technology Center, \\ 112 Thailand Science Park, Phahon Yothin Rd., Klong 1, Klong Luang, Pathumthani 12120 Thailand
}

${ }^{*}$ Corresponding author, e-mail: vitoon.sae@mahidol.ac.th

Received 15 Feb 2013

Accepted 26 Mar 2013

\begin{abstract}
The vertical jump is an essential skill used in a volleyball match but the information on the principal muscles used is incomplete. The present study aimed to identify the major muscles used in vertical jump by employing the principal component analysis (PCA) and the analysis of area under the curve (AUC). Ten elite female volleyball players (NV) and 10 female sedentary controls (SC) participated in this study. Each subject performed 2 jumping styles: squat jump and vertical stop jump. Electrical activities from thirteen muscles in the arm, trunk, and leg were recorded with electromyography (EMG). EMG data were processed using PCA and AUC methods, and the major muscles were identified by the first mode of PCA and the highest AUC. Our results showed that the PCA method was more sensitive than the AUC method for classifying the group differences in major muscles during jumping. Distinct activation of the erector spinae may explain the better performance in the NV compared to the SC, who preferentially used other trunk muscles in jumping. Our observation indicates that the PCA method is an appropriate analytical tool to differentiate the major muscles used in jumping between elite athletes and sedentary controls. This finding provides important information for designing training programmes especially for vertical jumps in sports.
\end{abstract}

KEYWORDS: PCA, electromyography, squat jump, vertical stop jump

\section{INTRODUCTION}

A vertical jump is a complex movement that requires a coordination of several muscles in the trunk, arms, and legs. This type of movement is the key component frequently used during blocking and spiking in a volleyball game ${ }^{1}$, and could contribute significantly to the match success. From a clinical perspective, the ability to perform a vertical jump is a reliable indicator of the lower limb power which has been validated across a wide range of populations ${ }^{2,3}$. In addition, the measurement of vertical jump is recommended to be used for predicting levels of mobility and the risk of falls in the elderly ${ }^{4}$. There are two vertical jumping styles, squat jump (SJ) and vertical stop jump (VSJ). The former is a form of jumping without countermovement starting from stationary, semi-squatting position without arm swing and no countermovement of the lower legs 5 . The latter, on the other hand, is a form of jumping with countermovement where a person runs a few steps before landing on both legs and jumps with preparatory countermovement ${ }^{6}$. The countermovement of arm swing with rapid flexion of hip, knee, and ankle joints in VSJ before jumping commonly known as stretch shortening cycle creates larger joint moments at the start of taking off 6,7 .

Previous studies indicated that the primary muscles contributing to $\mathrm{SJ}$ jumping performance are the knee extensor muscles ${ }^{8-10}$. In contrast, the primary muscles in VSJ are not only knee extensors but also ankle plantar flexors that provide the breaking force before taking off ${ }^{6,11}$. Furthermore, the momentum from running, countermovement, and the use of arm swing are required for successful VSJ jumping. Several studies indicated that stretch-shortening cycle and countermovement of lower limb muscles generated additional benefits on VSJ jumping performance, resulting in higher vertical jump compared to $\mathrm{SJ}^{7,9,12}$. The use of arm swing also builds up an extra-energy for increasing the velocity of the take-off allowing the person to attain larger joint momentum useful for vertical jump ${ }^{13,14}$. Although there are several mechanisms that operate together to enhance the jumping performance, muscle function is a key factor because 
of its ability to create the upward force to pull the trunk up. To date, it is still unclear which muscle plays the most significant role in producing effective jumping. Several previous investigations focused their studies on the muscles of lower extremities ${ }^{8-11}$. However, the act of vertical jump requires the whole body participation. Hence the information regarding the contributions of arm and trunk muscles is lacking, even though these muscles seem to play an important part in the vertical jump.

The principal component analysis (PCA) is a mathematical method that applies a matrix operation called singular value decomposition and the least square approximation principle. In clinical biomechanics, PCA has been used to eliminate a large number of highly interrelated variables leaving a small number of independent factors ${ }^{15,16}$. Several studies ${ }^{17,18}$ applied the PCA method to identify those variables playing a major role in jumping. It has been suggested that the time element of jumping is more important than the force element ${ }^{17}$ and the increased duration of take-off can produce higher jump than the increase of vertical force in volleyball players ${ }^{18}$. However, none of the previous research employed the PCA method to quantify muscle recruitments in vertical jump. This information can be useful for planning specific muscles training in order to achieve ultimate jumping performance. Hence in this study, PCA method was selected as the approach to identify the main muscles that contribute mostly to jumping performance. The sensitivity of the PCA method was also compared with the common EMG analysis of area under the curve (AUC). We hypothesized that the PCA method would be more sensitive than the analysis of AUC in identifying the major muscles of vertical jump.

\section{METHODS AND SUBJECTS}

\section{Participants}

The experimental protocol in the present study was approved by the Centre of Ethical Reinforcement for Human Research at Mahidol University (MUIRB 2008/114.1507). Twenty female subjects aged between 18 and 26 years participated in this study. Ten volleyball players (NV) were recruited from the national team of Thailand. Ten sedentary control subjects (SC), who were higher than $165 \mathrm{~cm}$ and have never undergone volleyball training, were college students. The subjects in both groups were age-matched. All subjects had no history of serious injuries of both lower extremities, such as fracture or rupture of ligament or tendon, at least 6 months before
Table 1 Characteristics and training experience in the national volleyball players (NV) and sedentary control (SC).

\begin{tabular}{lrc}
\hline $\begin{array}{l}\text { Characteristics and } \\
\text { training experience }\end{array}$ & NV & SC \\
\hline Age $(\mathrm{yr})$ & $22.9 \pm 2.9$ & $20.9 \pm 3.1$ \\
Weight $(\mathrm{kg})$ & $66.0 \pm 8.5$ & $55.5 \pm 9.1^{*}$ \\
Height $(\mathrm{cm})$ & $177.1 \pm 6.3$ & $169.4 \pm 1.7^{*}$ \\
BMI $\left(\mathrm{kg} / \mathrm{m}^{2}\right)$ & $21.0 \pm 1.3$ & $19.3 \pm 2.9$ \\
Playing duration (yr) & $11.9 \pm 4.4$ & $0^{*}$ \\
Time in volleyball team $(\mathrm{yr})$ & $4.1 \pm 3.3$ & $0^{*}$ \\
Training frequency (h/week) & $28.7 \pm 6.1$ & $0^{*}$ \\
\hline
\end{tabular}

Values are mean \pm standard deviation.

* Significant difference at $p<0.05$.

testing. None of them have hip, knee or ankle joint operation. All subjects signed an informed consent before participating in the study. Each subject was required to pass the triple-hop distance test, which was used as the screening test for lower limb dynamic balance test in athletes ${ }^{19}$. The general characteristics of the subjects are shown in Table 1.

\section{Testing protocols}

Each subject was asked to wear spandex shorts and running shoes. After shaving and cleaning the skin, the electromyography electrodes were attached on thirteen targets muscles on the right side, including anterior deltoid (AD), posterior deltoid (PD), biceps brachii (BB), triceps brachii (TB), latissimus dorsi (LD), erector spinae (ES), rectus abdominis (RA), vastus medialis (VMO), rectus femoris (RF), biceps femoris $(\mathrm{BF})$, semitendinosus (ST), tibialis anterior (TA), and gastrocnemius (MG). The Telemyo EMG system (Telemyo 2400T, Noraxon, Inc., USA) was used to capture the EMG data at the sampling rate of $1500 \mathrm{~Hz}$. Maximum voluntary contraction (MVC) was obtained from individual tested muscles ${ }^{20}$. A force plate (Kistler type $9281 \mathrm{CA}$; Kistler Instrument Inc., Switzerland) was used to measure the ground reaction force at $1000 \mathrm{~Hz}$ to identify the phase of jumping. A yard stick was placed beside the force plate to measure the vertical jump height. Prior to jumping, the standard reach height was obtained from each individual by measuring the maximum distance that a person in the upright standing position lifted the left hand as high as possible to touch the yard stick.

All subjects were asked to perform two jumping styles; squat jump (SJ) and vertical stop jump (VSJ), in a random sequence. All jumping styles were double leg jumps on the force platform. The 'jumping reach height' was measured as the maximum distance on the yard stick that each subject touched with the left 

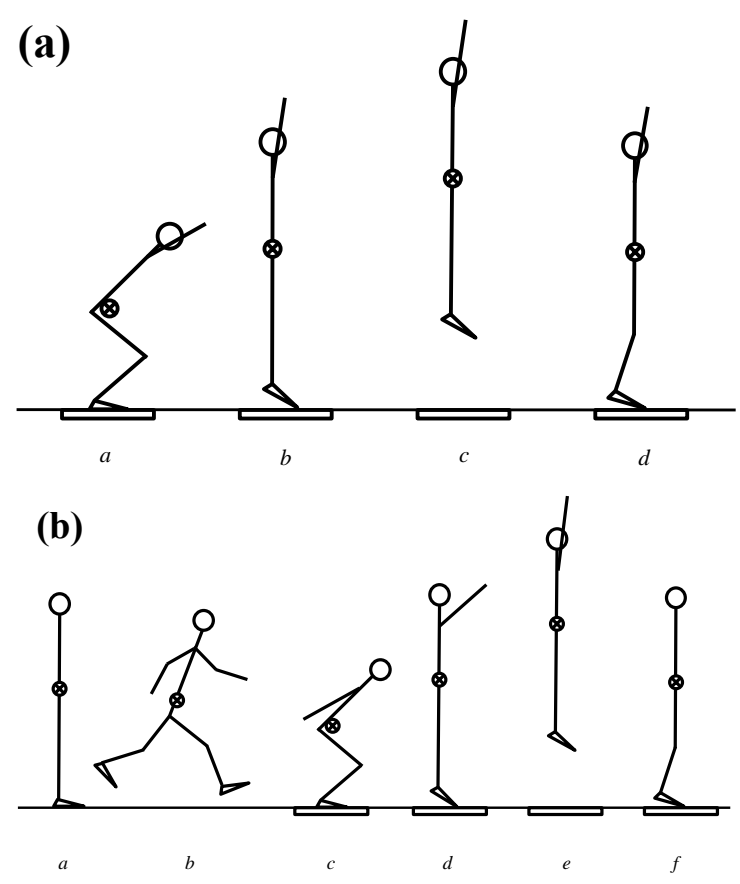

Fig. 1 Sequence of actions in (a) squat jump: ' $a$ ' semisquat on the force plate while raising left arm, ' $b$ ' taking off, ' $c$ ' jumping as high as possible, and ' $d$ ' landing; and (b) vertical stop jump: ' $a$ ' starting at 2 steps away from force plate, ' $b$ ' running 2 steps, ' $c$ ' double legs landing on force plate, ' $d$ ' taking off, ' $e$ ' jumping as high as possible with countermovement, and ' $f$ ' landing.

hand when jumping as high as possible. The "vertical jump height' was then obtained by subtracting the 'standing reach height' from the 'jumping reach height'. Practice trials were allowed for the subjects to become familiarized with the jumping tasks.

After jumping, the 'vertical jump height' was used to calculate for the lower limb power by using the following equation ${ }^{21}$ :

$$
P=\left(2.21 \mathrm{~W} \mathrm{~kg}^{-1} \mathrm{~m}^{-1 / 2}\right) W_{\mathrm{t}} \sqrt{d},
$$

where $P$ is the power, $W_{\mathrm{t}}$ is the body weight in jumping clothes, and $d$ is the vertical jump height. The constant $\left(2.21 \mathrm{~W} \mathrm{~kg}^{-1} \mathrm{~m}^{-1 / 2}\right)$ is based upon the rate of falling bodies.

In SJ (Fig. 1a), each subject was instructed to initially stand on the mark placed in the middle of the force plate in the semi-squatting position for 3$5 \mathrm{~s}$ before jumping as high as possible without countermovement of the arm and leg 22 . In VSJ (Fig. 1b), each subject was instructed to start at 2 steps away from the force plate. Each subject began by running from the starting point and stop when both legs have landed on the mark placed on the force plate before jumping with 2 legs on the centre marker before jumping ${ }^{9}$. Both types of jumping were performed using the individuals' preferred technique and no other instructions regarding jumping technique were given to the subjects to avoid instruction effect on their natural performances.

Each subject performed 5 trials to get at least 3 successful trials of vertical jump task with a 15 -s interval rest period between trials to prevent fatigue ${ }^{23}$. The two jumping styles, SJ and VSJ, were performed in a random order.

\section{Data analysis}

The data from the trial that showed the maximum vertical jump height were analysed using MATLAB. The vertical ground reaction force data were filtered through the 4th order Butterworth filter at $25 \mathrm{~Hz}$. These data were then used for dividing the phase of jumping ${ }^{11}$. The propulsion phase of each jumping style was identified as the duration from the changes in ground reaction force from the baseline to the time when both feet were lifted off the force plate.

All data from EMG were filtered with 4th order of Butterworth filter before further analysis. The AUC, which has been used as an indicator of work done by the muscle 24,25 , was calculated by the integration of EMG data during the propulsion phase using the $100 \mathrm{~Hz}$ filtered and rectified EMG. Subsequently, the AUC of each muscle was normalized by its own AUC during maximal voluntary contraction (MVC). The AUC data of each muscle are presented as the percentage value of MVC.

For the PCA method, raw EMG data were filtered at $25 \mathrm{~Hz}$ and normalized with their MVC before analysing for the major component. The covariance matrix calculates were computed with the help of MATLAB routines. In each subject, the data matrix $D$ was created, whose row represented the 13 muscles and column represented the normalized EMG response in time. The PCA analysis of these data set was straight forward by computing the singular value decomposition of the matrix $D=U \Sigma V^{*}$, where $\Sigma$ is a rectangular diagonal matrix with non-negative diagonal entries, $U$ and $V^{*}$ are unitary matrices, and ${ }^{*}$ represents the conjugate transpose. The $i$ th (spatial) PCA mode was represented by the $i$ th column of matrix $U$. The domination of each PCA modes was determined by comparing its corresponding diagonal entry in matrix $\Sigma$. The major muscles during different jumping styles from PCA analysis were quantified as the muscles that had the highest absolute magnitudes ranging between zero and one. The magnitude for 

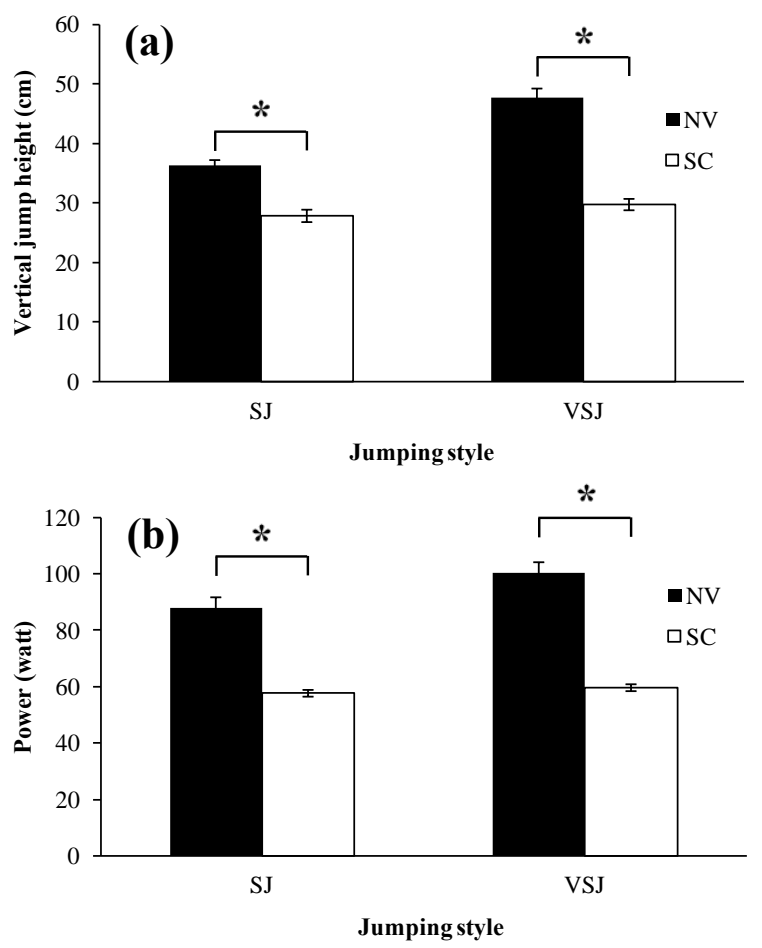

Fig. 2 (a) Vertical jump height and (b) lower limb power of national volleyball players (NV) and sedentary controls (SC) during SJ and VSJ. *: Significant difference at $p<0.05$.

a particular muscle that is close or equal to zero means the contribution from that particular muscle is negligible in that PCA mode. In this study, only the first PCA mode which covered 55\% of all variances was reported. The first two muscles that demonstrated the highest absolute magnitudes of each jump were counted. The frequency of these muscles were summarized across subjects and reported as major muscles for each jumping style.

Statistical analysis was performed using SPSS version 17.0. Means and standard errors of the means were calculated from descriptive statistics. Differences between two groups were determined by using an independent sample $t$-test at significant level of 0.05 .

\section{RESULTS}

The vertical jump height and lower limb power are shown in Fig. 2. The jump height and lower limb power of athlete $(\mathrm{NV})$ were significantly higher than those of sedentary control (SC) in both SJ and VSJ jumping styles.

The AUC of SJ and VSJ are shown in Fig. 3. In SJ (Fig. 3a), erector spinae (ES), vastus medialis
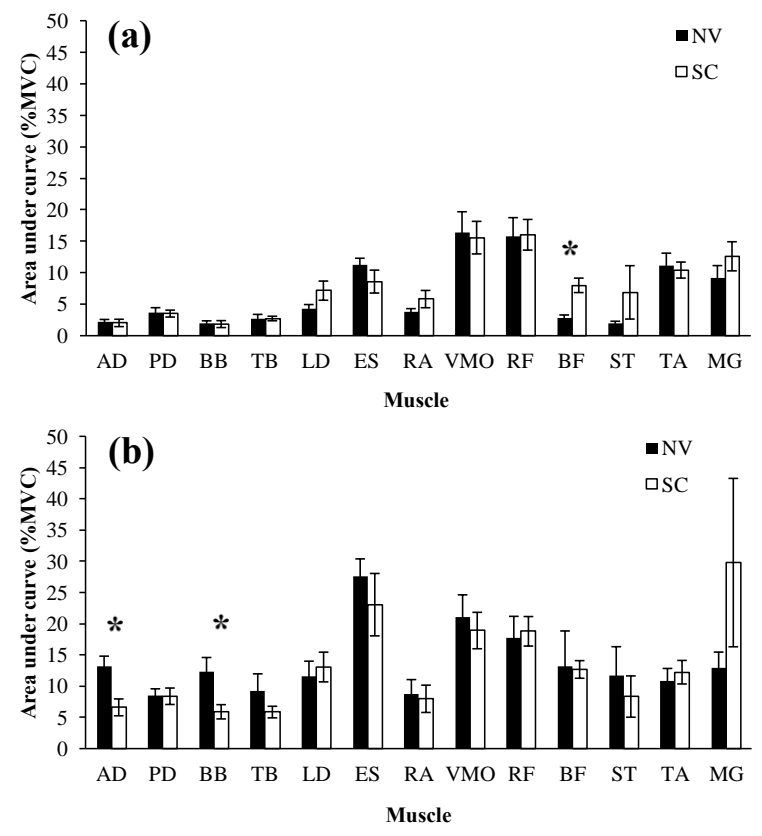

Fig. 3 AUC of the muscles in national volleyball players (NV) and sedentary controls (SC) during (a) squat jump and (b) vertical stop jump. AUC of each muscle was normalized to its own maximum voluntary contraction. Values are means $\pm \mathrm{SE}$. Muscles tested were: anterior deltoid (AD), posterior deltoid (PD), biceps brachii (BB), triceps brachii (TB), latissimus dorsi (LD), erector spinae (ES), rectus abdominis (RA), vastus medialis oblique (VMO), rectus femoris (RF), biceps femoris $(\mathrm{BF})$, semitendinosus (ST), tibialis anterior (TA), and medial gastrocnemius (MG). $*$ : Significant difference at $p<0.05$.

(VMO), rectus femoris (RF), tibialis anterior (TA), and medial gastrocnemius (MG) showed greater recruitments than the other muscles. Interestingly, SC demonstrated a greater recruitment of biceps femoris (BF) than NV did, whereas the recruitments of other muscles was not significantly different between the two groups. On the other hand, the AUC analysis of VSJ showed that all of the observed muscles were recruited during this jumping style (Fig. 3b). Of these muscles, erector spinae (ES), vastus medialis (VMO), and rectus femoris (RF) were among the muscles showing greatest recruitments. When the major muscles used for this performance by $\mathrm{NV}$ and SC were compared, only the anterior deltoid (AD) and biceps brachii $(\mathrm{BB})$ were activated in the $\mathrm{NV}$ group more than the SC group.

Results of PCA method are shown in Figs. 4 and 5. The major muscles of squat jump (SJ) in the NV group (Fig. 4a) were latissimus dorsi (LD), erector 

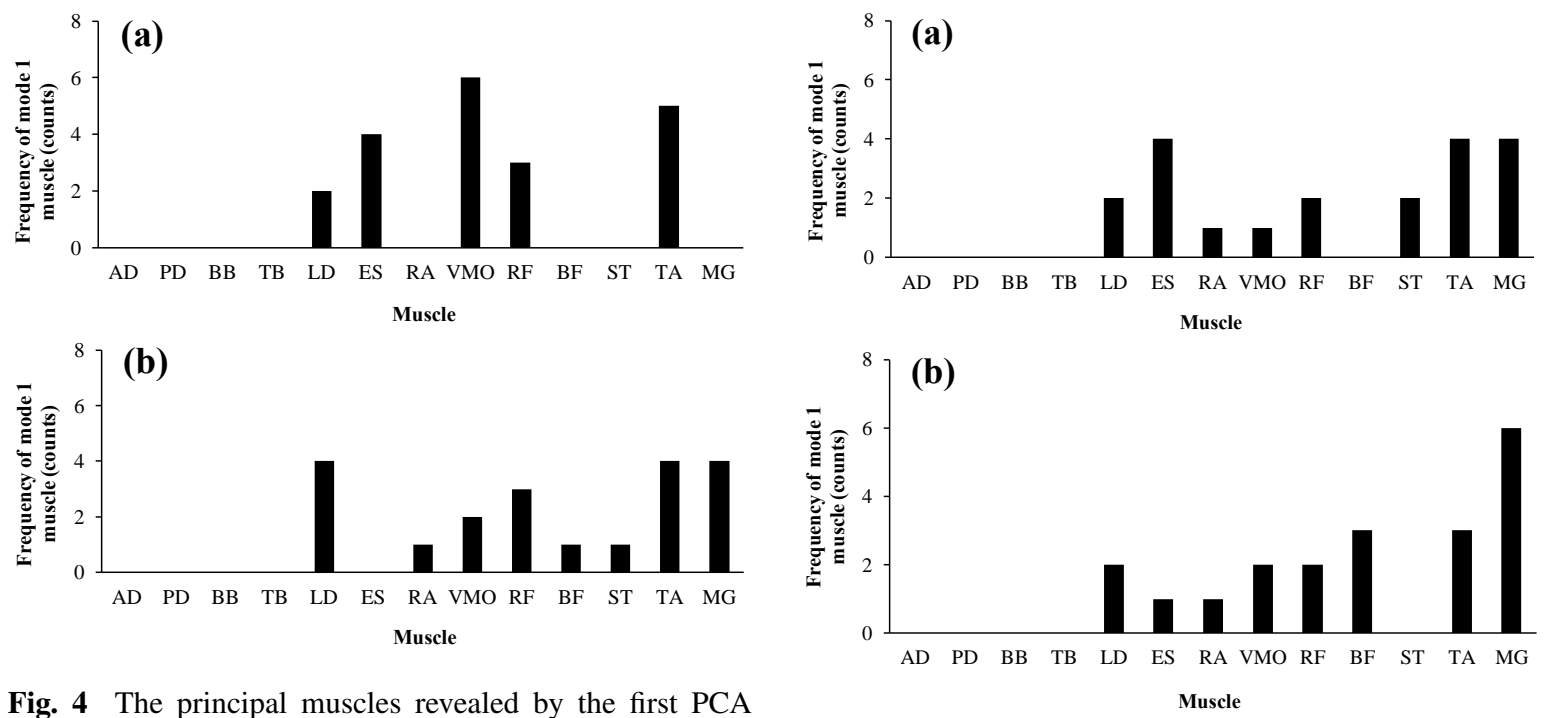

Fig. 4 The principal muscles revealed by the first PCA mode in squat jump performed by (a) national volleyball player and (b) sedentary control. Muscles tested were: anterior deltoid (AD), posterior deltoid (PD), biceps brachii (BB), triceps brachii (TB), latissimus dorsi (LD), erector spinae (ES), rectus abdominis (RA), vastus medialis oblique (VMO), rectus femoris (RF), biceps femoris (BF), semitendinosus (ST), tibialis anterior (TA), and medial gastrocnemius (MG).

spinae (ES), vastus medialis (VMO), rectus femoris (RF), and tibialis anterior (TA). These findings corresponded to the results from the analysis of AUC shown in Fig. 3a with the exception of latissimus dorsi (LD) and gastrocnemius (MG). In addition, PCA method revealed differences in several major muscles performed by SC compared to NV, including erector spinae (ES), rectus abdominis (RA), biceps femoris (BF), semitendinosus (ST), and medial gastrocnemius (MG) (Fig. 4b). These differences were more pronounced in the PCA method compared to the analysis by AUC.

The major muscles used by NV during vertical stop jump (VSJ) analysed by PCA method were latissimus dorsi (LD), erector spinae (ES), rectus abdominis (RA), vastus medialis (VMO), rectus femoris (RF), semitendinosus (ST), tibialis anterior (TA), and medial gastrocnemius (MG) (Fig. 5a). It was evident that the PCA method could differentiate a specific number of major muscles contributing to VSJ compared to the analysis of AUC (Fig. 3b). In addition, PCA analysis identified the differences between $\mathrm{NV}$ and $\mathrm{SC}$ in the activities of the erector spinae (ES), biceps femoris (BF), semitendinosus (ST), and medial gastrocnemius (MG) during VSJ.

Fig. 5 The principal muscles revealed by the first PCA mode in vertical stop jump performed by (a) national volleyball player and (b) sedentary control. Muscles tested were: anterior deltoid (AD), posterior deltoid (PD), biceps brachii (BB), triceps brachii (TB), latissimus dorsi (LD), erector spinae (ES), rectus abdominis (RA), vastus medialis oblique (VMO), rectus femoris (RF), biceps femoris (BF), semitendinosus (ST), tibialis anterior (TA), and medial gastrocnemius (MG).

\section{DISCUSSION}

The present study has demonstrated that the PCA method is more sensitive than the analysis of AUC in identifying the major muscles used in vertical jump, especially during vertical stop jump. Moreover, when the comparison between athlete and novice volleyball players was made, PCA method was proven to be highly appropriate in detecting subtle differences between subject groups. In contrast, the AUC analysis could not provide sufficient group comparison, even though the jumping performances (jump height and power) were significantly different between groups.

The AUC was calculated from the integration of EMG signal over a particular time normalized to its signal at maximum voluntary effort. This variable has been used as an indicator of the total work done by the muscle over a period of time ${ }^{24,25}$. The AUC value reflects the amount of muscle recruitment such that the higher AUC amplitude the larger recruitment of that muscle. However, the comparison among different muscles has been limited because the analysis of AUC does not allow true comparison between muscles. Nevertheless, AUC may be used to approximate the 
relationship among different muscles when the task is not complicated (as in SJ) such that the results from AUC analysis and PCA method are not much different. However, when the task is more complex (as in vertical stop jump), AUC analysis cannot provide the accurate comparison. On the other hand, the PCA is computed in such a way that all input variables are calculated together so that the interrelationship between different muscles can be directly evaluated ${ }^{15,17}$. Hence the PCA is more precise in identifying which muscles significantly contribute to the jumping performance.

In this study, it has been shown that latissimus dorsi, erector spinae, vastus medialis, rectus femoris and tibialis anterior are the major muscles involved in SJ. The roles of vastus medialis and rectus femoris in extending the knees during SJ are commonly reported in the previous studies and the strength of these two muscles has been suggested as a predictor of jump height ${ }^{8-10}$. Unlike the other studies, our study detected the important roles of latissimus dorsi, erector spinae and tibialis anterior. We believed that latissimus dorsi may not play a major role in the generation of the jumping force but it was activated to stabilize the arms beside the trunk during jumping. The activation of erector spinae creates the compressive force on the lumbar vertebrae which could enhance the vertebral stability allowing effective arm and leg movements 26,27 . The role of tibialis anterior was suggested by Spagele and colleagues ${ }^{11}$ to counter the action of plantar flexion as this muscle was activated at the latter phase of jumping. Also, the tibialis anterior may be involved in the preparation of the body prior to the force generation by gastrocnemius. A previous study examining the action of rising to toe showed that the activation of tibialis anterior occurred prior to the gastrocnemius $^{28}$. The authors concluded that early activation of tibialis anterior was considered as the anticipatory postural adjustments that were operated through the feed forward circuit. During normal standing, the body weight falls on the foot towards the heel. The anticipatory action of tibialis anterior serves to move the body weight from the heel to toe so that the heel would be able to lift from the ground and the toes could be used as the fulcrum for propelling the body upward. Hence the role of tibialis anterior as a principal muscle found during SJ would involve the preparation of the body so that the gastrocnemius can function effectively.

The PCA method clearly depicted the differences between the athletes and sedentary groups. In the SJ, athlete groups selected the erector spinae, which is the trunk extensor, whereas the sedentary groups pre- ferred the rectus abdominis, which is the trunk flexor. The trunk extensors are the antigravity muscles and their activation during taking off could help to propel the body upward against the gravity, whereas the trunk flexors could have the opposite effect, pulling the body downward. The choice of trunk muscle selection in the sedentary control group could result in an ineffective jumping which may lead to the lower leg compensation; the assistance of BF-ST (hip extensors) and MG (plantar flexors) in addition to the VMORF (knee extensors). Hence it could be interpreted that the sedentary group required additional assistance from the hip extensors to propel the body upward as a result of using the inappropriate trunk muscles or the lack of sufficient lower limb muscle strength to perform jumping.

During the vertical stop jump, latissimus dorsi, erector spinae, rectus abdominis, vastus medialis, rectus femoris, semitendinosus, tibialis anterior, and medial gastrocnemius are significant contributors to this jumping style. The roles of vastus medialis and rectus femoris are similar to those in the SJ. The coupling action of tibialis anterior and gastrocnemius has been suggested to absorb reaction force, especially when stopping with two legs before taking off ${ }^{6}$. Tibialis anterior also plays an important role during VSJ as it works with gastrocnemius to absorb lower limb force during landing. At the time the running stops (before jumping), the anterior shear force of proximal tibia is maximized since the ankle joint moves in the anterior direction with rapid deceleration followed immediately by a vertical jump ${ }^{6,9}$. Previous studies suggested that during the countermovement jump, the reinforcement from the arm swing and rapid knee flexion is required ${ }^{13,14}$ and this fits the role of both latissimus dorsi and semitendinosus. Erector spinae not only provides trunk stability but also works to extend the trunk in the acceleration phase of jumping to upright the body to increase jumping performance ${ }^{26,27}$. Differences between the athlete and sedentary groups during the vertical stop jump were similar to those during the SJ. The use of erector spinae was dominant in the athlete group, while the sedentary group preferred the medial gastrocnemius. The preference of ST in athletes and BF in sedentary controls is still unclear and may be due to different knee positions at the initial take off.

Our findings emphasized the importance of trunk extensors namely erector spinae in order to obtain the optimal jumping performance. The training of trunk extensors, either selective muscle or functional strength training, should be incorporated into the volleyball training. Furthermore, shoulder and knee 
muscles were equally important to achieve higher jump. Hence these muscle groups should also receive special training. Our suggestion is in accordance with the previous studies that developed the volleyball training program by training the whole body, such as run-up speed, lower limb strength and power training, and arm swing training ${ }^{1,10,24}$. It should be noted that the observation from this study was based on one athletic population that underwent volleyball training. Thus it is unknown whether our findings are limited only to volleyball or generally applicable to other types of sports. Further studies would be required to elucidate this issue.

\section{CONCLUSIONS}

Principal component analysis is the appropriate method to identify the major muscles contributing to jumping performance and the differences in muscle activations between athletes and sedentary controls. The selection of major muscles in athletes clearly different from the sedentary controls, especially the muscles in the trunk.

Acknowledgements: The authors wish to thank the volunteers of this study, the Sports Authority of Thailand and the Department of Physical Education, Ministry of Tourism and Sports. We are grateful to Prof. Chumpol Pholpramool and Assoc. Prof. Pipat Cherdrungsi for their valuable suggestions and editorial assistance. This study was supported by the Program Strategic Scholarships for Frontier Research Network for the PhD Programme from the Commission on Higher Education, Thailand.

\section{REFERENCES}

1. Ham DJ, Knez WL, Young WB (2007) A deterministic model of the vertical jump: implications for training. J Strength Condit Res 21, 967-72.

2. Rittweger J, Schiess1 H, Felsenberg D, Runge M (2004) Reproducibility of the jumping mechanography as a test of mechanical power output in physically competent adult and elderly subjects. J Am Geriatr Soc 52, 128-31.

3. Farias DL, Teixeira TG, Madrid B, Pinho D, Boullosa DA, Prestes J (2013) Reliability of vertical jump performance evaluated with contact mat in elderly women. Clin Physiol Funct Imag 33, 288-92.

4. Runge M, Rittweger J, Russo CB, Schiessl H, Felsenberg D (2004) Is muscle power output a key factor in the age-related decline in physical performance? A comparison of muscle crosee section, chair rising test and jumping power. Clin Physiol Funct Imag 24, 225-340.

5. Bobbert MF, Casius LJ, Sijpkens IW, Jaspers RT (2008) Humans adjust control to initial squat depth in vertical squat jumping. J Appl Physiol 105, 1428-40.
6. Chappell JD, Creighton RA, Giuliani C, Yu B, Garrett WE (2007) Kinematics and electromyography of landing preparation in vertical stop-jump: risks for noncontact anterior cruciate ligament injury. Am J Sports Med 35, 235-41.

7. Nagano A, Komura T, Fukashiro S, Himeno R (2005) Force, work and power output of lower limb muscles during human maximal-effort countermovement jumping. J Electromyogr Kinesiol 15, 367-76.

8. de Ruiter CJ, Vermeulen G, Toussaint HM, de Haan A (2007) Isometric knee-extensor torque development and jump height in volleyball players. Med Sci Sports Exerc 39, 1336-46.

9. Bradley PS, Olsen PD, Portas MD (2007) The effect of static, ballistic, and proprioceptive neuromuscular facilitation stretching on vertical jump performance. $J$ Strength Condit Res 21, 223-6.

10. Earp JE, Joseph M, Kraemer WJ, Newton RU, Comstock BA, Fragala MS, et al (2010) Lower-body muscle structure and its role in jump performance during squat, countermovement, and depth drop jumps. J Strength Condit Res 24, 722-9.

11. Spagele T, Kistner A, Gollhofer A (1999) Modelling, simulation and optimisation of a human vertical jump. J Biomech 32, 521-30.

12. Sheppard JM, Dingley AA, Janssen I, Spratford W, Chapman DW, Newton RU (2011) The effect of assisted jumping on vertical jump height in highperformance volleyball players. J Sci Med Sport 14, 85-9.

13. Lees A, Vanrenterghem J, De Clercq D (2004) Understanding how an arm swing enhances performance in the vertical jump. J Biomech 37, 1929-40.

14. Hara M, Shibayama A, Takeshita D, Fukashiro S (2006) The effect of arm swing on lower extremities in vertical jumping. $J$ Biomech 39, 2503-11.

15. Tabachnik BG, Fidell LS (2007) Using Multivariate Statistics, 5th edn, Pearson Education, Boston.

16. Daffertshofer A, Lamoth CJ, Meijer OG, Beek PJ (2004) PCA in studying coordination and variability: a tutorial. Clin Biomech 19, 415-28.

17. Kollias I, Hatzitaki V, Papaiakovou G, Giatsis G (2001) Using principal component analysis to identify individual differences in vertical jump performance. Res $Q$ Exerc Sport 72, 63-7.

18. Laffaye G, Bardy BG, Durey A (2007) Principal component structure and sport-specific differences in the running one-leg vertical jump. Int J Sports Med 28, $420-5$.

19. Hamilton RT, Shultz SJ, Schmitz RJ, Perrin DH (2008) Triple-hop distance as a valid predictor of lower limb strength and power. J Athletic Training 43, 144-51.

20. Hislop HJ, Montgomery J, Connolly BH (1995) Daniels and Worthingham's Muscle Testing: Techniques of Manual Examination, 5th edn, WB Saunders, Philadelphia.

21. Adams G, Beam W (2008) Exercise Physiology: Lab- 
oratory Manual, 5th edn, McGraw-Hill, USA.

22. Bobbert MF (2001) Dependence of human squat jump performance on the series elastic compliance of the triceps surae: a simulation study. J Exp Biol 204, 533-42.

23. Pereira G, Almeida AG, Rodacki AL, Ugrinowitsch C, Fowler NE, Kokubun E (2008) The influence of resting period length on jumping performance. J Strength Condit Res 22, 1259-64.

24. Winter D (2009) Biomechanics and Motor Control of Human Movement, 3rd edn, John Wiley \& Sons, USA.

25. Richards J (2008) Biomechanics in Clinic and Research: an Interactive Teaching and Learning Course, Churchill Livingstone/Elsevier, China.

26. Shinkle J, Nesser TW, Demchak TJ, McMannus DM (2012) Effect of core strength on the measure of power in the extremities. J Strength Condit Res 26, 373.

27. Willardson JM (2007) Core stability training: Applications to sports conditioning programs. J Strength Condit Res 21, 979.

28. Nardone A, Schieppati M (1988) Postural adjustments associated with voluntary contraction of leg muscles in standing man. Exp Brain Res 69, 469-80. 